\title{
A Basic Study on Stimulation Pulse Width Determination for Dynamic Presentation of Electrocutaneous Sensation Patterns
}

\author{
Yuka MINEGISHI ${ }^{\dagger}$, Nonmember, Takashi WATANABE ${ }^{\dagger \dagger a)}$, Member, \\ and Makoto YOSHIZAWA ${ }^{\dagger \dagger \dagger}$, Nonmember
}

\begin{abstract}
SUMMARY In this study, we aimed to realize a method of presenting dynamic electrocutaneous sensation patterns as a user interface for persons with disabilities. In this study, the previously used pulse width $(200 \mu \mathrm{s})$ was used in pattern recognition testing on the forearm and was compared with the short pulse width $(80 \mu \mathrm{s})$ used for stimulation. A cathodic pulse with a frequency of $100 \mathrm{pps}$, which was suggested to be suitable in our previous study, was used. Experimental results on neurologically intact subjects suggested that the previously used pulse width was suitable and that a short pulse width would not be effective for stabilizing the stimulation amplitude or for improving the pattern recognition ability of our method. key words: electrocutaneous sensation, dynamic pattern presentation, pattern recognition
\end{abstract}

\section{Introduction}

In this study, we focused on a method of graphical tactile display using electrocutaneous sensation patterns for persons with visual or auditory impairments or as a new method of information presentation to assist rehabilitation. In our previous study [1], a method of dynamic pattern presentation was proposed, in which patterns were presented by moving the position of electrical stimulation on the forearm. The proposed method was found to enable the transmission of more information than the previous static pattern presentation method [2].

However, the parameters of stimulation pulse trains used for the dynamic pattern presentation were not examined sufficiently. The polarity of the stimulation pulse and the pulse frequency were tested in our previous experiments, in which a cathodic pulse with a frequency of 50 or 100 pps was suggested to be useful for presenting dynamic patterns [3]. On the other hand, only a pulse width of $200 \mu \mathrm{s}$ was used in our previous studies.

In this paper, electrical stimulation using a short pulse width $(80 \mu \mathrm{s})$ was examined to clarify whether or not the previously used pulse width $(200 \mu$ s) was suitable, because a short pulse width was considered to be effective for inducing an electrocutaneous sensation. That is, a shorter pulse width allows more spatially selective stimulation of nerve

\footnotetext{
Manuscript received December 2, 2008.

${ }^{\dagger}$ The author is with the Dept. Electrical and Communication Eng., Tohoku University, Sendai-shi, 980-8579 Japan.

${ }^{\dagger}$ The author is with the Dept. Biomedical Eng., Tohoku University, Sendai-shi, 980-8579 Japan.

${ }^{\dagger \dagger}$ The author is with the Cyberscience Center, Tohoku University, Sendai-shi, 980-8579 Japan.

a) E-mail: nabet@bme.tohoku.ac.jp

DOI: 10.1587/transinf.E92.D.1328
}

fibers [4]. It also reduces variations in the current threshold [5].

\section{Experimental Method}

A short pulse width $(80 \mu \mathrm{s})$ was tested in comparison with the previously used pulse width $(200 \mu \mathrm{s})$ in a pattern recognition experiment on six neurologically intact subjects (3 male and 3 female) using the seven presentation patterns shown in Fig. 1. The patterns were presented on the forearm as shown in Fig. 2 using the electrode array consisting of 9 electrodes that was designed in our previous study [1]. The stimulation time when presenting a pattern was $0.96 \mathrm{~s}$, which was performed by stimulating three electrodes in a sequence.

A total of four experimental sessions were performed on two consecutive days for two pulse width conditions in the following order:

1st day: 1) $80 \mu \mathrm{s}$, 2) $200 \mu \mathrm{s}$

2nd day: 1) $200 \mu \mathrm{s}, 2) 80 \mu \mathrm{s}$

A cathodic stimulation pulse with a frequency of $100 \mathrm{pps}$ was used under the same conditions as those in our previous

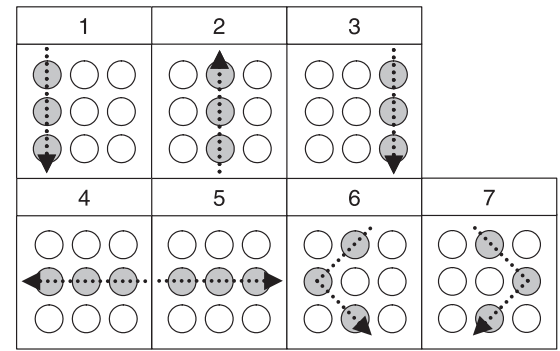

Fig. 1 Seven presentation patterns used in experiment.

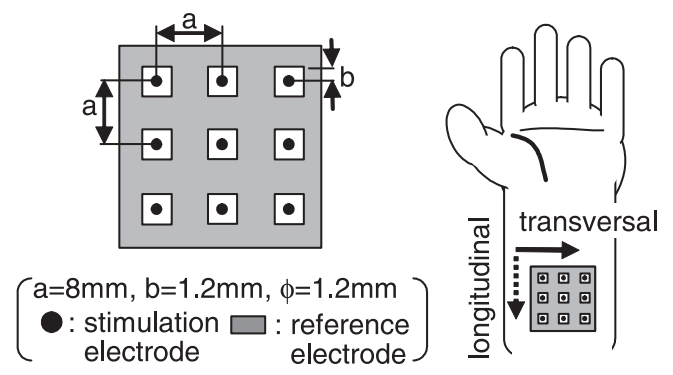

Fig. 2 Stimulation electrode array and stimulation position. 
study [1].

In each session, the stimulation pulse amplitude was first determined by each subject for each electrode as the value at which the sensation was comfortably experienced. Then, the subjects were subjected to a training stage. In this stage, one of the seven patterns was presented randomly and the correct pattern number was given to the subject after his/her answer. A total of 28 patterns were presented for the training. After the training stage, a pattern recognition test was carried out by presenting one of the seven patterns in random order. To make it difficult to predict the presentation pattern, the total number of presentations for each pattern was also randomized in each experimental session, during which the subjects had to identify a pattern 42 times.

\section{Results}

First, the coefficient of variation (CV) of the stimulation amplitudes was calculated for each session. In the calculation of $\mathrm{CV}$, the stimulation voltage amplitude for one electrode was removed in one session (subj.A, $80 \mu$ s (2nd)) because it was extremely large compared with those of the other eight electrodes. There was no major difference in the value of $\mathrm{CV}$ between the two pulse widths and between experimental sessions $(0.21 \pm 0.05$ for the short pulse width and $0.20 \pm 0.04$ for the long pulse width). Figure 3 shows the rate of change of $\mathrm{CV}$, calculated by

$$
\frac{C V(80 \mu \mathrm{s})-C V(200 \mu \mathrm{s})}{C V(200 \mu \mathrm{s})},
$$

where $C V(80 \mu \mathrm{s})$ and $C V(200 \mu \mathrm{s})$ denote $C V$ for pulse width of $80 \mu \mathrm{s}$ and $200 \mu \mathrm{s}$, respectively. The value of CV decreased in 5 out of 12 experiment days. However, no clear decrease in the value of CV for a short pulse width was observed in this result.

Figure 4 shows the correct recognition ratio (CRR) in each session in order of experiments. Figure 5 shows the rate of change of CRR, calculated by

$$
\frac{C R R(80 \mu \mathrm{s})-C R R(200 \mu \mathrm{s})}{C R R(200 \mu \mathrm{s})}
$$

where $C R R(80 \mu \mathrm{s})$ and $C R R(200 \mu \mathrm{s})$ denote CRR for pulse

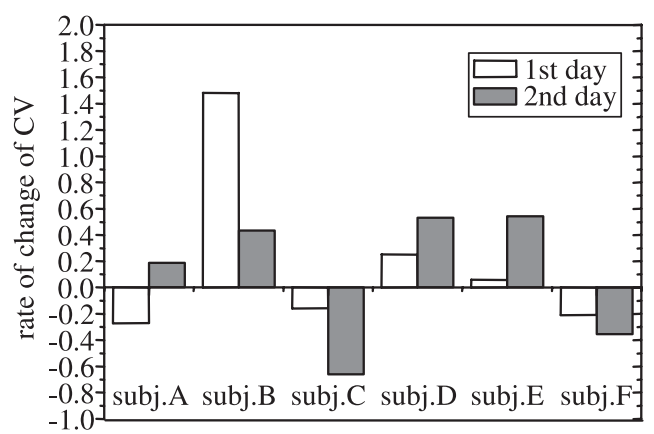

Fig. 3 Rate of change of coefficient of variation (CV) of stimulation amplitudes. widths of $80 \mu$ s and $200 \mu \mathrm{s}$, respectively. There was no correlation between CRR and the appearance ratio for all the subjects $(r=0.125 \pm 0.08)$. As shown in Fig. 5, CRR was improved by using the short pulse width on 3 out of 12 experiment days. However, by comparing the results of the two sessions in each day, Fig. 4 suggests that subjects A, C and D had a larger CRR for a $200 \mu$ s pulse width, subjects $B$ and $E$ had a larger CRR in the second session in each day, and subject $\mathrm{F}$ had a larger CRR in the first session in each day. That is, it is not clear that the value of CRR improved using the short pulse width.

\section{Discussion}

There is no difference in the average value of $\mathrm{CV}$ of the stimulation amplitude between different pulse widths and no clear decrease in CV upon using a short pulse width. In addition, no clear improvement of CRR by using a short pulse width was observed. From these results, it is considered that there is no advantage in using a short pulse width in our method. A pulse width longer than $200 \mu$ s was not tested in this study, because it might decrease the pain threshold and the dynamic range, defined as the set of values between the threshold amplitude and maximum amplitude [6].

Possible reasons why the shorter pulse width did not reduce the variation of stimulation amplitude are as follows. First, the stimulation amplitude was determined by each subject as the value at which he/she could comfortably

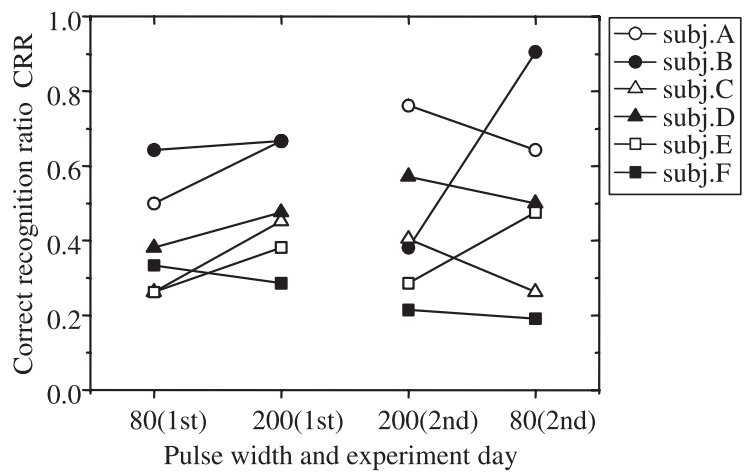

Fig. 4 Correct recognition ratio (CRR) in each session in order of experiments.

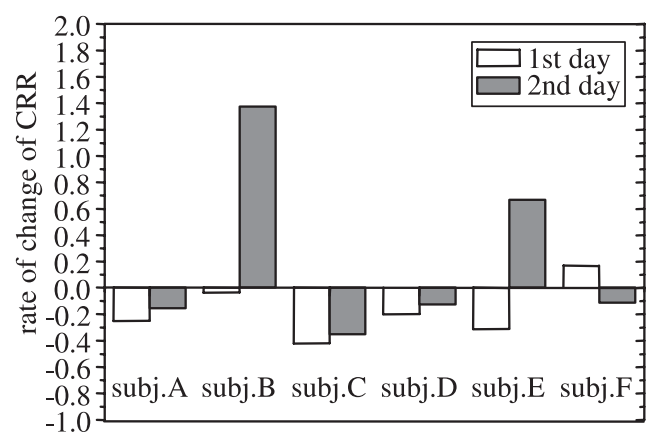

Fig. 5 Rate of change of correct recognition ratio (CRR). 
detect the sensation. Second, the voltage stimulation amplitude is discussed in this study but the variation of the threshold for current stimulation was investigated in the previous study [5]. Third, a shorter pulse width has been suggested to increase the dynamic range [4].

The reasons why there was no difference in the value of CRR between different pulse widths are not clear. However, an increased stimulation amplitude may cause a decrease in CRR because it activates nerve fibers over a large area. In all the subjects, ratios of the average stimulation amplitude of the nine electrodes with a pulse width of $80 \mu \mathrm{s}$ to that with a width of $200 \mu$ s were almost 1.0 or higher. This was because the subjects determined the amplitude of which the sensation was detected comfortably. Therefore, it is possible that the increased stimulation pulse amplitude reduced the effect of the short pulse width on improving the spatial selectivity of activating nerve fibers.

As seen in our previous experiment [1], it was possible to improve CRR by repeating the recognition experiment. In this study, the subjects were trained before each recognition test. Although training might improve CRR, half the subjects did not have a value of CRR larger than $50 \%$ in most sessions. Subjects A and B, who had higher values of CRR, participated in our previous study [3]. Although subject C participated in our previous study [1], the value of CRR was not high. Subjects D, E and F participated in our experiments for the first time. By considering the results for various subjects, it is suggested that a difference in pulse width does not affect the pattern recognition ability because none of the subjects showed a clear improvement in the value of CRR.

\section{Conclusion}

In this paper, pattern recognition experiments using different pulse widths were performed to determine whether or not the previously used pulse width $(200 \mu$ s) was suitable. For our dynamic pattern presentation method, it was suggested that the previously used pulse width was satisfactory and that a short pulse width would have no effect on reducing the variation of stimulation voltage and on improving pattern recognition ability. An effective training method is necessary to improve the pattern recognition ratio for practical applications of dynamic pattern presentation using electrocutaneous sensation.

\section{Acknowledgement}

This work was supported in part by the Ministry of Education, Culture, Sports, Science and Technology of Japan under a Grant-in-Aid for Scientific Research (B).

\section{References}

[1] Y. Sato, T. Watanabe, M. Yoshizawa, and N. Hoshimiya, "Feasibility study of transmission of information by presenting dynamic cutaneous sensation patterns elicited by surface electrical stimulation," J. Soc. of Biomechanisms, vol.31, no.1, pp.30-35, 2007.

[2] K.A. Kaczmarek and S. Haase, "Pattern identification as a function of stimulation current on a fingertip-scanned electrotactile display," IEEE Trans. Neural. Syst. Rehabil. Eng., vol.11, no.3, pp.269-275, 2003.

[3] Y. Minegishi, T. Watanabe, and M. Yoshizawa, "Basic experiments of physiological properties for information presenting method using dynamic electrocutaneous sensation patterns," Int. J. Bioelectromagn., vol.9, no.1, pp.30-31, 2007.

[4] W.M. Grill, Jr. and J.T. Mortimer, "The effect of stimulus pulse duration on selectivity of neural stimulation," IEEE Trans. Biomed. Eng., vol.43, no.2, pp.161-166, 1996.

[5] H. Kajimoto and S. Tachi, "Sensation stabilization of large area, dense electro-tactile display," Proc. 20th Ann. Symp. Biological and Physiological Eng., pp.243-244, 2005.

[6] K.A. Kaczmarek, J.G. Webster, P. Bach-y-Rita, and W.J. Tompkins, "Electrotactile and vibrotactile displays for sensory substitution systems," IEEE Trans. Biomed. Eng., vol.38, no.2, pp.1-16, 1991. 